\title{
Prevalencia y factores asociados a la venta de cigarros a menores de edad en el Distrito Federal
}

\author{
Pablo Antonio Kuri-Morales, M en C,(I) Mario Cortés-Ramírez, M en C,(1) \\ Patricia Cravioto-Quintana, Dra en $C^{(1)}$
}

\author{
Kuri-Morales PA, Cortés-Ramírez M, Cravioto-Quintana P. \\ Prevalencia y factores asociados a la venta de cigarros a \\ menores de edad en el Distrito Federal. \\ Salud Publica Mex 2005;47:402-4I2.
}

\begin{abstract}
Resumen
Objetivo. Conocer la prevalencia y los factores asociados a la venta de cigarros a menores en el Distrito Federal. Material y métodos. Estudio transversal realizado mediante una encuesta a expendedores de comercios que venden cigarros. Se generó un marco muestral mediante el SCINCE 2000 del INEGI, se seleccionaron probabilísticamente dos Areas Geoestadísticas Básicas (AGEB) por delegación y se realizó un censo de dichos comercios. Resultados. Se visitaron 577 comercios de los cuales $73 \%$ vendió cigarros a menores, $58 \%$ vendía cigarros sueltos; $2.6 \%$ preguntó la edad y I. $2 \%$ solicitó una identificación. De los expendedores, $51 \%$ fueron hombres, $37 \%$ cursó la secundaria, $57 \%$ había fumado, $42 \%$ tenía hijos menores y $99 \%$ sabía que está prohibido vender cigarros a menores. El análisis multivariado permitió establecer un modelo explicativo, y se encontró que cuando el expendedor es hombre o se venden cigarros sueltos se incrementa entre 2 y 5 veces la posibilidad de venta, respectivamente. Conclusiones. La venta de cigarros a menores en el Distrito Federal es independiente de las características del expendedor y del comercio.
\end{abstract}

Palabras clave: prevalencia; venta de cigarros a menores; Distrito Federal; México
Kuri-Morales PA, Cortés-Ramírez M, Cravioto-Quintana P. Prevalence and risk factors related to sale of cigarettes to minors in stores in Mexico City. Salud Publica Mex 2005;47:402-4I2.

\section{Abstract}

Objective. To identify the prevalence of the sale of cigarette to minors in stores in Mexico City. Material and Methods. A cross-sectional study was done applying a questionnaire to salespersons in stores that sell cigarettes. The SCINCE 2000 from INEGI was used to select and sample two Basic Geostatistical Areas (AGEB) per Delegation and a census of stores was carried out. Results. A total of 577 stores were visited, $73 \%$ sold cigarettes to minors, $58 \%$ sell loose cigarettes, only $2.6 \%$ asked the buyer's age, and I.2\% requested identification.Among the salespersons, $51 \%$ were men, $37 \%$ had secondary school studies, $57 \%$ were smokers, $42 \%$ had children underage, and $99 \%$ knew that the sale of cigarettes to minors is prohibited. The multivariate analysis established an explicative model and it was discovered that when the salesperson was male or if the cigarettes were sold individually the probability of sale was 2 and 5 times higher respectively. Conclusions. The sale of cigarettes to minors in Mexico City is not associated with the personal characteristics of the salespersons or with the stores.

Key words: prevalence; sale of cigarettes to minors; Mexico City; Mexico
E n México y otros países, la accesibilidad de los menores de edad a los productos derivados del tabaco -fundamentalmente a los cigarros- es un factor primordial que interactúa con otros factores psicosociales, como la aceptación, la falta de identidad o la presión de los pares, los cuales favorecen en los menores de edad el inicio del consumo de dichos productos y la adicción al tabaco. Debe señalarse que la venta de cigarros a población menor de 18 años está prohibida en México desde 1984; a pesar de ello y de que es uno

(I) Dirección General de Epidemiología, Secretaría de Salud.

Fecha de recibido: 25 de enero de 2005 - Fecha de aprobado: 25 de agosto de 2005 Solicitud de sobretiros: M en C. Mario Cortés Ramírez. Francisco de P. Miranda \# 177, 3er piso. Col. Unidad Lomas de Plateros, C.P. 01480 , México, D.F., México. Correo electrónico: marioc@dgepi.salud.gob.mx 
de los factores más influyentes en el acceso que tienen los menores a los derivados del tabaco, ${ }^{2}$ algunos establecimientos no cumplen con dicha disposición ${ }^{1}$ y la venta se lleva a cabo.

El presente trabajo, además de evaluar la prevalencia actual de la venta de cigarros a menores de edad en los comercios de la Ciudad de México, identifica los factores que se relacionan en el proceso, con el propósito de modificar las estrategias para eliminar o reducir esta venta y, en consecuencia, abatir el consumo de tabaco entre este grupo de edad pues está comprobado que una vez iniciado el consumo los rasgos farmacológicos de los componentes del tabaco, especialmente la nicotina, favorecen el desarrollo del tabaquismo, el cual fue definido en 1984 por la Organización Mundial de la Salud (OMS) como una forma de dependencia. En específico, se trata de una entidad clínica definida como adicción a la nicotina del tabaco que se manifiesta a través de la tolerancia, la dependencia y el síndrome de abstinencia. Se expresa por la necesidad compulsiva de consumir tabaco y la dificultad para abandonarlo, y constituye una forma de dependencia como la que se manifiesta en otras adicciones. ${ }^{3}$

Resulta evidente que, por sí sola, la información de que el consumo de tabaco produce efectos adversos en la salud humana es insuficiente para generar cambios a gran escala en el comportamiento social respecto a su consumo. Para abatir con éxito este problema se necesita una estrategia que englobe tanto aspectos sanitarios como aspectos psicológicos, sociales, económicos y políticos, y que permita comprender y modificar el contexto del tabaquismo en un sentido favorable a la salud. Es innegable que el control del tabaquismo está dificultado por dos cuestiones clave: su carácter adictivo y los intereses económicos asociados a la producción y la distribución de productos de la planta de tabaco. ${ }^{3}$

En nuestro país se ha identificado que el tabaquismo entre niños y adolescentes es un problema en ascenso, como lo muestran los datos de las encuestas nacionales de adicciones. Por ejemplo, la proporción de fumadores que iniciaron el consumo de tabaco antes de los 18 años presenta una tendencia creciente, que pasa de $52 \%$ en 1988 a $58.2 \%$ en 2002 , con un máximo de $61.4 \%$ en 1998 . Un problema adyacente es que el tabaquismo en los adolescentes actúa como puerta de entrada para el consumo de otras drogas.

En relación con la venta de cigarros a menores, en 1999 se publicaron los resultados del estudio comparativo de la venta de cigarros a menores en tres ciudades de la frontera entre México y Estados Unidos de América, donde se determinó que el porcentaje de vendedores dispuestos a vender cigarros a menores fue $6.1 \%$ en las Cruces, $18 \%$ en El Paso y $98.1 \%$ en Ciudad
Juárez. ${ }^{4}$ Para la Ciudad de México sólo existe la evaluación realizada en 1997, donde se encontró que 79\% de los intentos de compra de cigarros por menores fueron exitosos. ${ }^{1}$

\section{Material y métodos}

El presente estudio se llevó a cabo mediante un diseño transversal, en el cual se registró la información del resultado del intento de compra de cigarros por un menor en un formato prediseñado, y a través de las observaciones hechas en cada comercio, así como de la aplicación de un cuestionario a los expendedores de los mismos. La fase operativa del proyecto se realizó en cuatro etapas:

- El levantamiento del marco muestral,

- el intento de compra de cigarros por menores de 18 años de edad,

- la observación de las características de los comercios, $y$

- la entrevista al expendedor.

\section{Muestra y marco muestral}

El tamaño de la muestra se calculó considerando la proporción de venta encontrada en el estudio realizado en la Ciudad de México en $1997^{1}$ y el valor del efecto de diseño utilizado en estudios de adicciones, definido como "la pérdida o la ganancia por el efecto de conglomerar elementos de la población para formar unidades muestrales", con una tasa de "No respuesta" de $15 \%$ y una confianza de $95 \%$, lo cual dio un resultado de 570 comercios requeridos.

Debido a que no se cuenta con un registro actualizado de los comercios que expenden cigarros en la Ciudad de México, para obtener una muestra aleatoria representativa se realizó el siguiente esquema de muestreo:

- Se llevó a cabo un muestreo probabilístico por conglomerados, utilizando la información del Sistema de Consulta para Información Censal (SCINCE, 2000) del Instituto Nacional de Estadística, Geografía e Informática (INEGI).

- Se seleccionaron por medio de muestreo aleatorio simple dos Areas Geoestadísticas Básicas (AGEB) por cada delegación política, considerando al AGEB como "el área geográfica que constituye la unidad básica del marco geoestadístico y que se encuentra delimitada por detalles geográficos permanentes y fácilmente identificables".

- Se obtuvieron los croquis de cada una de las 32 AGEB seleccionadas a través del SCINCE 2000. 
- Se realizó un censo para obtener un listado actualizado de todos los comercios que venden cigarros en cada una de las AGEB seleccionadas.

\section{Intento de compra y observaciones}

Para el intento de compra, previamente se capacitaron equipos de trabajo formados cada uno por dos menores de entre 10 y 14 años y dos adultos, y se realizó lo siguiente:

- El menor ingresó al comercio (seleccionado previamente) e intentó comprar cigarros;

- momentos después, el primer adulto ingresó al comercio y observó las características del mismo solicitando y comprando cigarros sueltos cuando fue necesario. miento.

Este procedimiento se repitió en cada estableci-

\section{Entrevista al expendedor}

El segundo adulto del equipo regresó al comercio y entrevistó al expendedor con el que se realizó el intento de compra (el primero indicó de quién se trataba). Cabe señalar que la entrevista se llevó a cabo independientemente del resultado del intento de compra hecha por el menor.

\section{Definición de variables}

Variable dependiente:

- Venta de cigarros a menores

Variables independientes:

- Características del menor (edad, sexo)

- Características del expendedor (edad, sexo, escolaridad, estado civil, conocimiento de la prohibición de venta de cigarros a menores, si tiene hijos menores de edad, opiniones sobre vender a menores).

- Características del comercio (ubicación, tipo, accesibilidad a cigarros, presencia de cartel de "No venta a menores").

Criterios de inclusión, exclusión y eliminación:

- Inclusión: cualquier comercio ubicado en el D. F. que venda cigarros.

- Exclusión: cualquier comercio ubicado en el D.F. exclusivo para mayores de 18 años.
- Eliminación: cuando el comercio, a juicio del responsable del equipo, sea inseguro para el menor (que presente personas ingiriendo bebidas alcohólicas o cualquier manifestación de violencia).

\section{Consideraciones éticas}

De acuerdo con los artículos 96100 y 102 de la Ley General de Salud, este estudio se cataloga como de riesgo nulo para los participantes. Sin embargo, para garantizar los derechos de los menores y obtener explícitamente la autorización por parte de los padres o tutores, se les solicitó carta de consentimiento de participación; asimismo, se les otorgó la libertad de dar por terminada su participación en el momento que lo desearan. Cabe mencionar que previamente se les otorgó a los menores material informativo acerca de los efectos del consumo de tabaco en la salud; asimismo, se impartieron pláticas conjuntas a padres y a menores sobre el problema del tabaquismo.

\section{Resultados}

\section{Marco muestral}

En las 32 AGEB seleccionadas se identificaron 617 comercios en los que se vendían cigarros, independientemente del tipo de establecimiento. Por criterios de inclusión y exclusión, nueve quedaron fuera del estudio. Finalmente el tamaño de muestra se redujo a 608 comercios; sin embargo, no fue posible llevar a cabo el operativo del intento de compra de cigarros por un menor en 31 comercios. Debido a lo anterior, el número de intentos de compra se redujo a 577, lo que significó una tasa de "No respuesta" de $5 \%$ para esta fase del estudio.

\section{Características de los comercios}

En relación con el tipo de comercios registrados en la muestra, la mayoría $(81.4 \%)$ fueron tiendas, misceláneas o comercios habilitados a través de las ventanas en casas habitación, seguidas de los puestos ambulantes o semifijos $(8.5 \%)$. Solamente se registró un restaurante en el cual las diferencias resultaron estadísticamente significativas, lo cual indica que existe una relación entre las AGEB de cada delegación y el tipo de comercio (cuadro I).

Al realizar la inspección visual de los comercios y evaluar la venta de cigarros sueltos, se encontró que sólo $21 \%$ de éstos presentaban anuncios de prohibición de venta de cigarros a menores de edad en lugares visibles a los consumidores; en $58 \%$ de estos establecimientos (334) se venden cigarros sueltos (cuadro I). 


\section{Intento de compra}

La prevalencia de la venta de cigarros a menores de edad en los comercios de la Ciudad de México encontrada en el 2002 fue de $73.1 \%$ (cuadro I). Asimismo, se encontró que $58 \%$ de los comercios presentan la modalidad de venta de cigarros por unidad.

La dinámica de la transacción fue similar en la mayoría de los comercios, y se observó que no se cumple con la disposición oficial de solicitar una identifi-

Cuadro I

Características del comercio y del intento de compra de cigarRos POR MENORES De EDAD en el Distrito Federal durante el 2002: información global y por Resultado de la venta

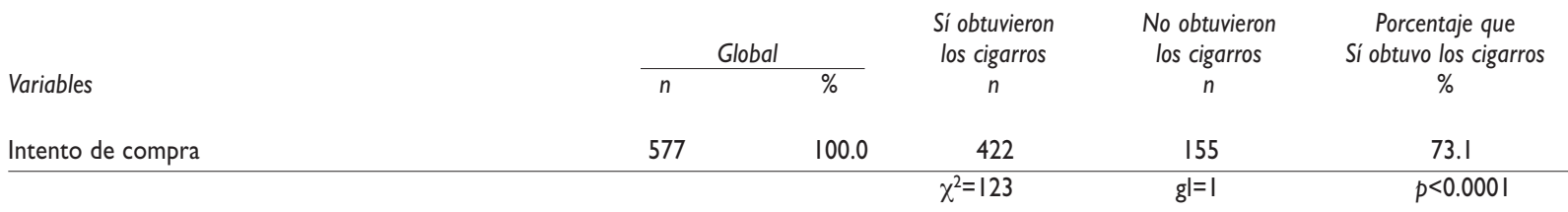

Tipo de comercio

\begin{tabular}{|c|c|c|c|c|c|}
\hline Supermercado & 5 & 0.9 & 3 & 2 & 60.0 \\
\hline Minisuper & 12 & 2.1 & 5 & 7 & 41.7 \\
\hline Tienda / ventana / miscelánea & 470 & 81.4 & 341 & 129 & 72.6 \\
\hline Farmacia & 19 & 3.3 & 15 & 4 & 78.9 \\
\hline Papelería & 10 & 1.7 & 6 & 4 & 60.0 \\
\hline Vinatería & II & 1.9 & 6 & 5 & 54.5 \\
\hline Ambulante / semifijo & 49 & 8.5 & 46 & 3 & 93.9 \\
\hline Restaurante & I & 0.2 & 0 & I & 0.0 \\
\hline$\chi^{2}=2529.4 \quad g \mid=7$ & $p<0.0001$ & & $\chi^{2}=23.1$ & $g \mathrm{~g}=7$ & $p=0.002$ \\
\hline
\end{tabular}

Presentan anuncios

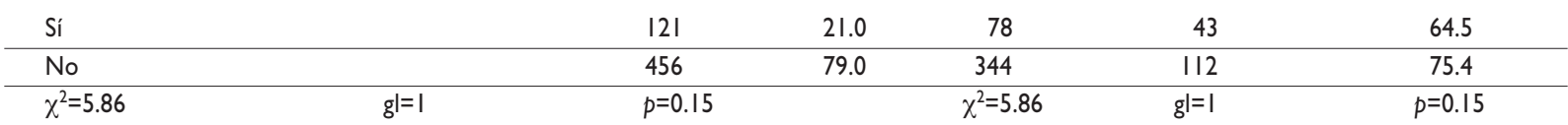

Venden cigarros sueltos

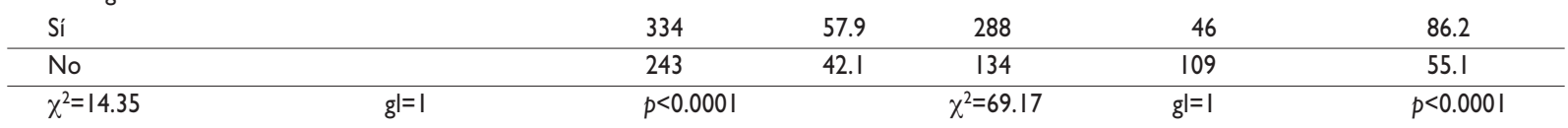

El expendedor solicitó identificación

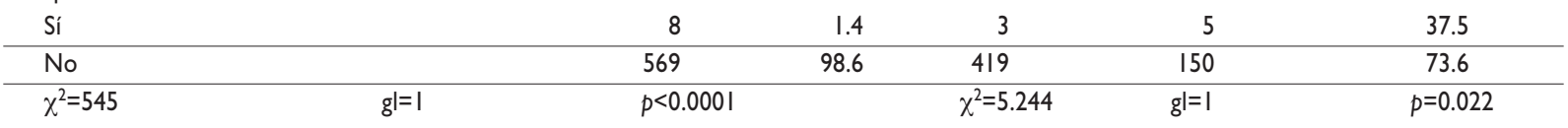

El expendedor preguntó la edad

\begin{tabular}{llrrrrrr} 
Sí & & 15 & 2.6 & 9 & 6 & & \\
\hline No & & 562 & 97.4 & $4 \mid 3$ & $\mid 49$ & \\
\hline$\chi^{2}=5 \mid 8$ & $g|=|$ & $p<0.000 \mid$ & & $\chi^{2}=\mid .353$ & $g|=|$ & $p=0.245$
\end{tabular}

El expendedor preguntó para quién eran los cigarros

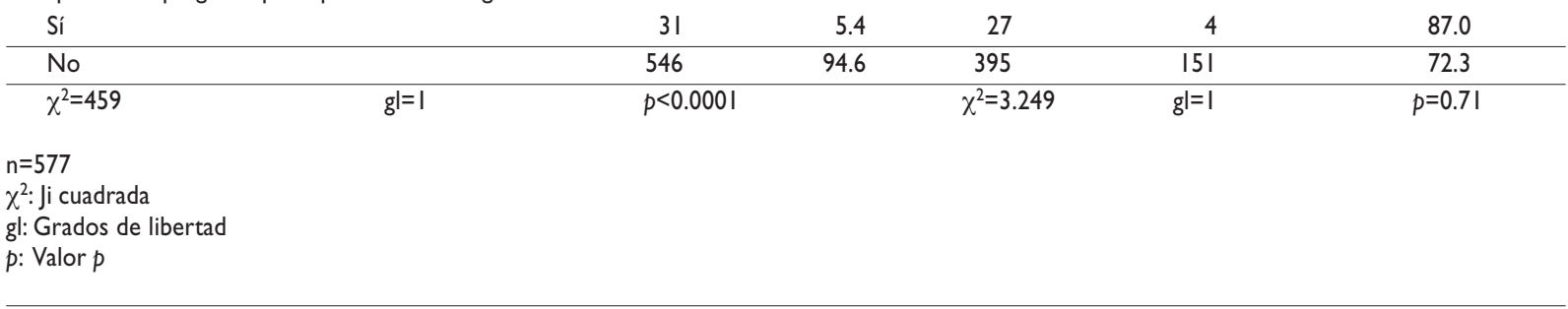


cación al menor al momento que éste solicita cigarros [esta acción ocurrió sólo en ocho ocasiones (1.4\%)]. En cuanto a las preguntas "sobre la edad del menor" y "para quién solicitaba los cigarros", en la gran mayoría de los casos (97.4\% y 94.6\%, respectivamente), el menor manifestó que no le realizaron ninguna pregunta al solicitar los cigarros en los comercios (cuadro I).

\section{Características del expendedor (global y por resultado de la venta)}

Se aplicó el cuestionario diseñado ex profeso a los expendedores con quienes se realizó el intento de compra de cigarros independientemente del resultado del mismo. También se intentó entrevistar al total de expendedores; sin embargo, 27 de ellos no aceptaron la entrevista y debido a esta negativa no se registró información sobre esta población. La falta de participación por parte de los expendedores significó una tasa de "No respuesta" de 5\% respecto al total de intentos de compra por los menores, lo cual indica que 550 expendedores proporcionaron información.

La distribución por sexo entre los expendedores fue prácticamente la misma (50.2\% hombres y $49.8 \%$ mujeres). En cuanto a la edad que manifestaron al momento de la entrevista, se registraron edades desde 10 hasta 80 años, con una media de $36.43 \pm 15.5$, lo que nos indica que la mayoría de esta población entra en la categoría de adultos jóvenes en edad económicamente activa. Para facilitar el manejo de los datos se agrupó la edad en quinquenios; se encontró un mayor porcentaje en el grupo etáreo de 35 y más años $(47.5 \%)$, seguido por el grupo de 25 a 29 años $(15.2 \%)$, y finalmente por el grupo de 10 a 14 años (cuadro II).

La escolaridad registrada entre los entrevistados tuvo representación en todos los niveles propuestos, con un mayor porcentaje en secundaria $(36.9 \%)$ y primaria $(29.6 \%)$. Cabe resaltar que $10.2 \%$ de los expendedores cuentan con grados de licenciatura o posgrado. Respecto al estado civil, casi $63 \%$ declaró mantener algún tipo de relación conyugal, mientras que los solteros representaron $30 \%$ de los casos. Cuando se preguntó al expendedor si tenía hijos menores de edad, más de la mitad $(57.6 \%)$ respondió que no. Con referencia al hábito tabáquico de los expendedores, en forma global se encontró que más de la mitad $(57 \%)$ ha fumado tabaco alguna vez, con una diferencia significativa en relación con los que no han fumado nunca (cuadro II).

Al hacer una comparación entre los expendedores que vendieron cigarros a menores y los que no lo hicieron, no se encontraron diferencias con sustento estadístico en la mayoría de las características. Entre las variables sociodemográficas únicamente el sexo presentó significancia estadística; asimismo, el hábito tabáquico entre los que vendieron y los que no vendieron presentó diferencias que no justifican validez estadística: $57.4 \%$ de los que vendieron han fumado, mientras que entre los que no vendieron $55.4 \%$ ha fumado (cuadro II).

\section{Opiniones y actitudes del expendedor respecto a la venta de cigarros a menores}

Cuando se cuestionó en forma directa al expendedor si vendía cigarros a menores, la gran mayoría (96.2\%) contestó categóricamente que no; sin embargo, su actitud respecto a la venta de cigarros a esta población fue contraria; es decir, la mayoría (72.2\%) de los que dijeron no vender habían vendido cigarros a menores antes de la entrevista (cuadro III).

En relación con la opinión de los expendedores al cuestionarles aspectos vinculados con la venta de cigarros a menores, se observó que casi todos (99.1\%) sabían que en México la venta de cigarros a menores está prohibida y manifestaron estar de acuerdo con ello (cuadro III). Sin embargo, nuevamente estas opiniones no fueron congruentes con el resultado de la venta, al registrarse que $73 \%$ de los que dijeron saber que en México vender cigarros a menores está prohibido, y que apoyan esta prohibición, vendieron cigarros a los menores del equipo antes de la entrevista.

Al preguntarles "Si no estuviera prohibida la venta de cigarros a menores, ¿usted les vendería?", la proporción de los que dijeron que no les venderían alcanzó $79.7 \%$; de ellos $70 \%$ había vendido cigarros a menores poco antes de la entrevista (cuadro III).

\section{Análisis (bivariado)}

Se investigó la relación que tienen algunas variables con la venta de cigarros a menores. Para las variables dicotómicas, esta asociación se calculó a través de la Razón de Momios de la Prevalencia $(R M p)$; en las variables politómicas se evaluó la relación a través de la prueba ji cuadrada.

En todas las delegaciones se registró la venta de cigarros a menores, la cual fluctuó entre $45.5 \%$ en la delegación Cuauhtémoc hasta $92.3 \%$ en Tlalpan; sin embargo, estas diferencias pueden deberse a las características de las AGEB en las que se realizó el operativo del intento de compra de cigarros por menores. El tipo de comercio también presentó relación con la venta de cigarros a menores, con un mayor porcentaje de venta, como era de esperarse, en los comercios semifijos o ambulantes $(93.9 \%)$. 


\section{Cuadro II}

Características sociodemográficas de los expendedores de comercios QUe Vendieron cigarros en el Distrito Federal dURANTE EL 2002: INFORMACIÓN GLOBAL Y POR RESULtAdO DE LA VENTA

\begin{tabular}{|c|c|c|c|c|c|}
\hline \multirow[b]{2}{*}{ Características del expendedor } & \multicolumn{2}{|c|}{ Global } & \multirow{2}{*}{$\begin{array}{c}\text { Sí vendió cigarros } \\
n\end{array}$} & \multirow{2}{*}{$\begin{array}{c}\text { No vendió cigarros } \\
n\end{array}$} & \multirow{2}{*}{$\begin{array}{c}\text { Porcentaje que } \\
\text { Si vendió cigarros } \\
\%\end{array}$} \\
\hline & $N$ & $\%$ & & & \\
\hline \multicolumn{6}{|l|}{ Sexo } \\
\hline Femenino & 274 & 49.8 & 188 & 86 & 68.6 \\
\hline Masculino & 276 & 50.2 & 215 & 61 & 78.0 \\
\hline$\chi^{2}=0.007$ & $p=0$. & & $\chi^{2}=6.053$ & $g \mid=I$ & $p<0.014$ \\
\hline
\end{tabular}

Edad

\begin{tabular}{lccccc}
10 a 14 años & 12 & 2.2 & 8 & 4 & 66.6 \\
\hline 15 a 19 años & 56 & 10.2 & 44 & 12 & 78.6 \\
\hline 20 a 24 años & 67 & 12.2 & 50 & 17 & 74.6 \\
\hline 25 a 29 años & 84 & 15.2 & 62 & 22 & 73.8 \\
\hline 30 a 34 años & 70 & 12.7 & 56 & 14 & 78.0 \\
\hline 35 y más años & $26 \mid$ & 47.5 & 183 & 78 & 70.1 \\
\hline$\chi^{2}=408.32$ & $p<0.0001$ & & $\chi^{2}=4.092$ & $g \mid=5$ & $p=536$
\end{tabular}

Escolaridad

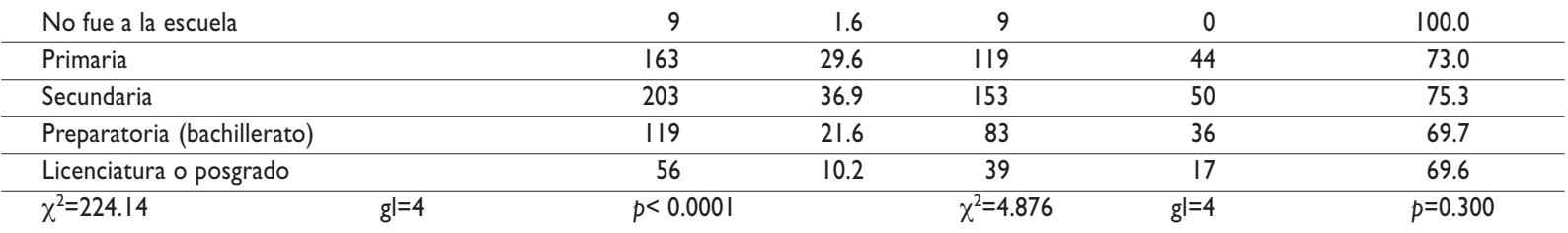

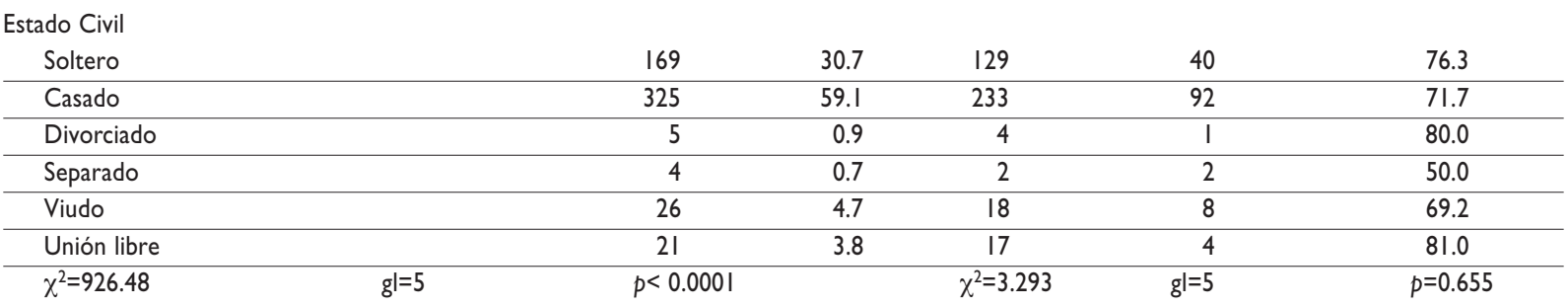

Tiene hijos menores de edad

\begin{tabular}{|c|c|c|}
\hline Sí & 42.4 & 0.8 \\
\hline No & 57.6 & 75.0 \\
\hline$\chi^{2}=12.82$ & & $=0.264$ \\
\hline
\end{tabular}

Ha fumado tabaco

\begin{tabular}{|c|c|c|c|c|c|c|}
\hline Sí & & 313 & 57.0 & 231 & 82 & 74.0 \\
\hline No & & 237 & 43.0 & 171 & 66 & 72.0 \\
\hline$\chi^{2}=10.502$ & $g \mid=1$ & $p=0.001$ & & $\chi^{2}=0.267$ & $g \mid=I$ & $p=0.605$ \\
\hline $\begin{array}{l}=550 \\
2: \text { Ji cuadrada } \\
\text { I: Grados de li } \\
\text { :Valor } p\end{array}$ & & & & & & \\
\hline
\end{tabular}

Las características significativas de los comercios, en relación con la venta a menores, fueron la presencia de anuncios de no venta a menores y el hecho de que en el comercio se vendieran cigarros sueltos $(R M p=1.66$ y 5.26, respectivamente) (cuadro IV).
Entre las variables del expendedor, sólo en el sexo se encontró que hay riesgo 1.6 veces de venta cuando el expendedor es hombre (IC 1.08-2.41, 95\%) (cuadro IV). Por otra parte, el hecho de que el expendedor manifestara no tener hijos menores de edad y el vivir con o sin 


\section{Cuadro III}

Opinión y actitudes del expendedor de comercios QUe venden cigarros en el Distrito Federal durante el 2002, RESPECTO A LA VENTA DE CIGARROS A MENORES DE EDAD: INFORMACIÓN GLOBAL Y POR RESULTADO DE LA VENTA

\begin{tabular}{ccccc} 
Global & & Sí vendió cigarros & No vendió cigarros & $\begin{array}{c}\text { Porcentaje que } \\
\text { Sí vendió cigarros }\end{array}$ \\
\hline & $\%$ & $n$ & $n$ & $\%$
\end{tabular}

¿Vende usted cigarros a menores?

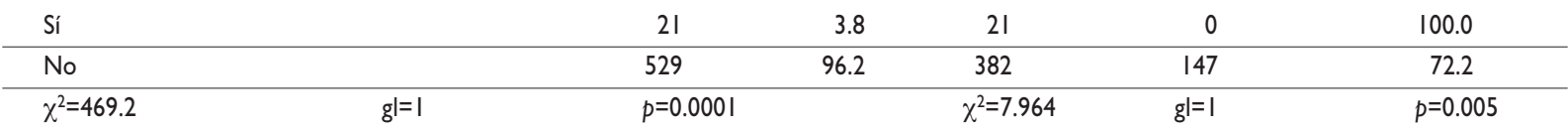

¿Sabe que está prohibida la venta de cigarros a menores de edad?

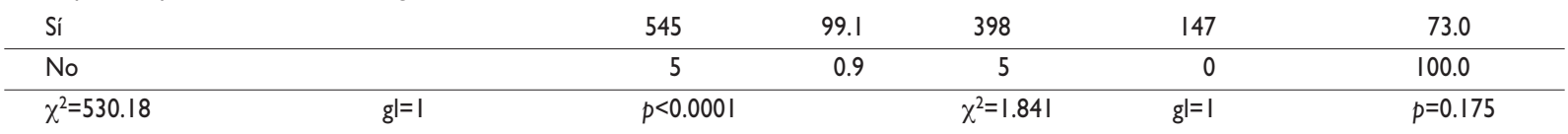

¿Está de acuerdo con esa prohibición?

\begin{tabular}{|c|c|c|c|}
\hline Sí & 398 & 146 & 73.0 \\
\hline No & 4 & 1 & 80.0 \\
\hline$\chi^{2}=529.18$ & $\chi^{2}=0.118$ & $g \mid=I$ & $p=0.731$ \\
\hline
\end{tabular}

Si la venta de cigarros a menores no estuviera prohibida, ¿usted les vendería?

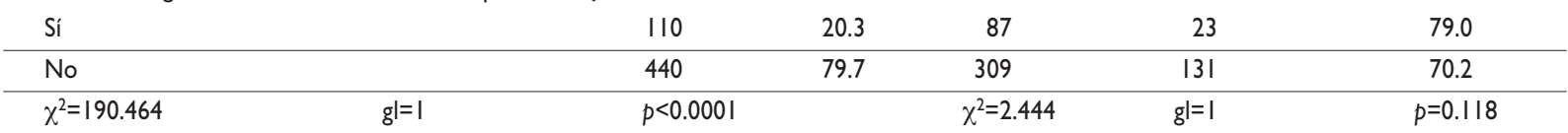

$\mathrm{n}=550$

$\chi^{2}$ : Ji cuadrada

gl: Grados de libertad

$p$ :Valor $p$

pareja, así como sus hábitos tabáquicos, no tuvo significancia estadística (cuadro IV).

\section{Análisis estratificado}

Se realizó un análisis estratificado con el propósito de evaluar una posible confusión entre las variables, controlando en forma individual por el sexo, el estado civil y la escolaridad del expendedor, así como por el tipo de comercio y la delegación en la que se encuentra ubicado.

$\mathrm{Al}$ controlar mediante estas variables se observó que ninguna de las variables estudiadas resultó confusora; es decir, las diferencias entre el riesgo crudo y el riesgo ajustado para cada una de ellas no fue mayor a $10 \%$.

\section{Análisis multivariado}

De acuerdo con el análisis previo, las variables que presentaron asociación significativa se introdujeron a diferentes modelos, mismos que fueron ejecutados utilizando la técnica backward. Los estadísticos empleados para la inclusión y la eliminación de las variables fueron la puntuación eficiente de Rao y el estadístico de Wald, respectivamente. En esta fase del análisis se utilizó el programa de cómputo SPSS ver. 10.0..$^{5-7}$

Se analizaron diversos modelos hasta encontrar el que mejor explicara la asociación entre la venta de cigarros a menores y las variables que fueron consideradas como de exposición. Por último, el modelo propuesto quedó integrado con las variables "delegación", "sexo del expendedor", "sexo del menor" y "venta de cigarros sueltos" (cuadro V).

Con base en lo anterior, se puede afirmar que el hecho de que un comercio venda cigarros sueltos incrementa el riesgo de que venda cigarros a menores de edad 5.4 veces (IC 3.4-8.7, 95\%), controlando por la delegación, el sexo del menor y el sexo del expendedor. Por otro lado, cuando el expendedor es hombre el riesgo de la venta a menores se incrementa 2.04 veces (IC 


\title{
Cuadro IV \\ Asociación entre La VENTA de CIgARROS A MENORES DE EDAD Y LAS CARACTERísticas del COMERCIO, Del expendedor y del intento de compra en el Distrito Federal durante el 2002
}

\author{
$\begin{array}{lll}R M p & \text { IC } 95 \% & \text { Valor } p\end{array}$
}

Variables del comercio

Dicotómicas

Tiene anuncios de "No venta de cigarros a menores"

Venden cigarros sueltos

Accesibilidad por autoservicio de cigarros sueltos

\begin{tabular}{ccc}
1.66 & $(1.04,2.65)$ & $<0.0001$ \\
5.26 & $(3.41,8.12)$ & $<0.0001$ \\
\hline 1.69 & $(0.46,7.28)$ & 0.400
\end{tabular}

Variables del expendedor

Dicotómicas

\begin{tabular}{|c|c|c|c|}
\hline Edad agrupada (hasta 17 años y de 18 en adelante) & 1.65 & $(0.74,3.65)$ & 0.2099 \\
\hline Sexo & 1.61 & $(I .08,2.4 I)$ & 0.0138 \\
\hline Tiene hijos menores de edad & 1.24 & $(0.83,1.85)$ & 0.2640 \\
\hline Ha fumado tabaco alguna vez & 1.11 & $(0.74,1.65)$ & 0.6050 \\
\hline Fuma actualmente & 1.15 & $(0.67,1.97)$ & 0.5790 \\
\hline Estado civil agrupado (vive con y sin pareja) & 1.15 & $(0.77, I .7 I)$ & 0.4820 \\
\hline
\end{tabular}

Variables del intento de compra

Dicotómicas

Preguntó el expendedor la edad al menor

296.25

$(31.86,2754.2)<0.0001$

Solicitó la identificación

666.67

$(47.53,21617)$

$<0.0001$

Preguntó para quién eran los cigarros

60.48

(6.5I, 56I.5)

$<0.0001$

RMp: Razón de momios para la prevalencia

IC $95 \%$ : Intervalo de confianza al $95 \%$

1.3-3.2, 95\%) controlando por la delegación, el sexo del menor y la venta de cigarros sueltos.

\section{Discusión}

La venta de cigarros a menores de edad es un hecho que presenta muchas aristas que es necesario abordar si se pretende eliminar el fenómeno. En esta investigación se consideraron tres causas necesarias para que se presente la venta: las características de los comercios, del expendedor y del comprador, que en conjunto integran la causa suficiente. Cabe señalar que, con las variables incluidas en el presente estudio, se logró conocer en forma general el panorama actual de este problema en la Ciudad de México.

Se realizó un análisis multivariado, donde se probaron variables referentes a las características de los comercios (aquéllas que presentaron asociación con significancia estadística); finalmente se llegó al modelo propuesto en el cuadro $\mathrm{V}$.

Estos resultados son de suma importancia, ya que nos indican que el alto porcentaje de venta encontrado
(73\%) no tiene relación con características específicas de los expendedores de comercios que venden cigarros. Lo anterior hace evidente que éstos anteponen la ganancia que les genera la venta de cigarros a menores de edad a la conciencia del daño que les pueden generar a los jóvenes.

Resulta alarmante que en $73 \%$ de los comercios seleccionados para el estudio se logrará obtener cigarros a través de menores de edad, lo cual hace evidente que este problema no ha sido abordado en forma adecuada o que las medidas empleadas no son aplicadas por no tener vigilancia de su cumplimiento. Asimismo, la venta de cigarros sueltos registrada en $58 \%$ de los comercios evidencia esa falta de vigilancia del cumplimiento de las disposiciones establecidas.

Setenta y tres por ciento de venta de cigarros a menores es uno de los más altos porcentajes que se han registrado, sólo superado por el encontrado en Ciudad Juárez ${ }^{4}$ que alcanzó $98 \%$, en el cual la metodología empleada fue similar a la del presente estudio. Asimismo, fue semejante al encontrado tanto en los vecindarios afroamericanos como en los barrios bajos 


\section{Cuadro V

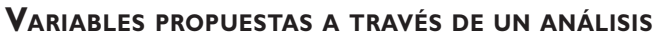 MULTIVARIADO PARA EXPLICAR LA VENTA DE CIGARROS A MENORES DE EDAD EN LOS COMERCIOS QUE VENDEN cigarros en el Distrito Federal durante el 2002}

\begin{tabular}{|c|c|c|c|c|}
\hline \multirow[b]{2}{*}{ Variable } & \multirow[b]{2}{*}{ B } & \multirow[b]{2}{*}{$\operatorname{Exp}(B)$} & \multicolumn{2}{|c|}{$\begin{array}{c}\text { IC } 95 \% \\
\text { Para exp (B) }\end{array}$} \\
\hline & & & INF & SUP \\
\hline \multicolumn{5}{|l|}{ Delegación } \\
\hline Tlapan & 3.988 & 53.964 & 5.674 & 513 \\
\hline Cuajimalpa & 2.229 & 9.286 & 2.525 & 34.2 \\
\hline Iztacalco & 2.004 & 7.415 & 1.534 & 35.8 \\
\hline Iztapalapa & I.44I & 4.226 & 1.074 & 16.6 \\
\hline Alvaro Obregón & 1.592 & 4.913 & 1.460 & 16.5 \\
\hline Gustavo A. Madero & 1.801 & 6.054 & 1.731 & 21.2 \\
\hline Xochimilco & 1.095 & 2.989 & 0.566 & 15.8 \\
\hline Tláhuac & 1.772 & 5.883 & 1.699 & 20.4 \\
\hline Venustiano Carranza & 1.179 & 3.251 & 0.987 & 10.7 \\
\hline Magdalena Contreras & 0.551 & 1.736 & 0.396 & 7.600 \\
\hline Azcapotzalco & 0.660 & 1.935 & 0.485 & 7.709 \\
\hline Milpa Alta & 0.770 & 2.161 & 0.660 & 7.075 \\
\hline Miguel Hidalgo & 0.985 & 2.679 & 0.798 & 8.994 \\
\hline Benito Juárez & 0.718 & 2.049 & 0.501 & 8.385 \\
\hline Coyoacán & 0.673 & 1.960 & 0.601 & 6.395 \\
\hline Sexo del menor (Masculino) & -0.521 & 0.594 & 0.324 & 1.089 \\
\hline Sexo del expendedor (Masculino) & 0.716 & 2.047 & 1.310 & 3.197 \\
\hline Venden cigarros sueltos & 1.695 & 5.449 & 3.398 & 8.738 \\
\hline Constante & -1.059 & 0.347 & & \\
\hline \multicolumn{5}{|c|}{$\begin{array}{l}\text { B: Valor beta } \\
\text { Exp (B):Valor del exponencial de beta } \\
\text { INF: Límite inferior del intervalo de confianza } \\
\text { SUP: Límite superior del intervalo de confianza }\end{array}$} \\
\hline
\end{tabular}

de blancos de Baltimore, donde las ventas fueron de $88.5 \%$ y $80.6 \%$, respectivamente. ${ }^{8}$ Cabe señalar que estos informes corresponden a zonas clasificadas como áreas de bajos ingresos, lo cual limita su comparación. Asimismo, en Ontario se alcanzó 27\%, ${ }^{9}$ donde el grupo de menores voluntarios tuvo un rango de edad de 13 a 18 años. Este rango fue diferente al que se utilizó en el presente estudio, que fue de menores de 10 a 14 años, lo que hace suponer que si se hubiera utilizado el mismo rango de edad, la diferencia en cuanto al porcentaje de venta habría sido mayor. Difranza en $1996^{10}$ registró 33\% en la ciudad de Massachussets, donde se evalúo la influencia del sexo, la edad y la presencia de maquinas expendedoras.

Con respecto a la distribución de comercios en la muestra, éstos presentaron variaciones importantes de acuerdo con la delegación donde se ubicaban como consecuencia de la selección aleatoria de las AGEB; esto es, se seleccionaron tanto áreas comerciales (cerca de mercados, de estaciones del metro o de paraderos de microbuses), donde el número de comercios fue muy alto, así como áreas residenciales muy exclusivas o algún conjunto habitacional de acceso restringido, en las cuales el número de comercios fue mínimo. Estas diferencias en la distribución por tipo de comercio en cada delegación pudo haber influido en los porcentajes de venta que se encontraron en cada una; sin embargo, la información con que se cuenta no permite establecer si la variación en los porcentajes de venta se debe a que en algunas delegaciones existe una mejor vigilancia sobre el cumplimiento de las disposiciones oficiales o es consecuencia de las características de la AGEB.

Como se esperaba, el número de comercios por tipo encontrado en todo el marco muestral tuvo un mayor porcentaje $(81.5 \%)$ de tiendas, ventanas o misceláneas. Esto puede observarse en la mayoría de las zonas que conforman al Distrito Federal, donde las tiendas son los establecimientos más numerosos en los que se venden cigarros, comparado con cualquier otro tipo de establecimiento (supermercados, minisuper... incluso vendedores ambulantes).

En relación con la exhibición de anuncios que prohiben la venta a menores, $21 \%$ los presentaban en lugares visibles al público. Cabe señalar que en algunos negocios se llegó a observar los carteles pero en lugares de difícil apreciación, sobre todo para los menores (lugares en alto o detrás de refrigeradores, por ejemplo), o éstos estaban detrás de carteles de promoción de otros productos. De acuerdo con el porcentaje encontrado, aparentemente existió un aumento de la proporción de comercios que presentaron anuncios de restricción de venta a menores en relación con lo reportado por Kuri y colaboradores en 1997 (11.8\%). Cabe señalar que en los puestos ambulantes no se observaron estos carteles.

La venta de cigarros por unidades (sueltos) surgió como una alternativa para disminuir la posible subestimación del resultado, pues la conducta del expendedor podría ser distinta ante la presencia obligada de una tercera persona adulta durante el intento de compra y la observación simultánea. En relación con esta modalidad de venta no se encontró en la literatura revisada algún antecedente al respecto; sin embargo, se cree que $58 \%$ de lo encontrado en este estudio está subestimado, ya que en muchas ocasiones en el operativo de campo, al solicitar cigarros sueltos, los expendedores dudaron o cambiaron de opinión negándolos, cuando parecía que los iban a surtir. Este cambio de actitud pudo ser reflejo de la observación y el desconocimiento que el expendedor tenía del comprador; muchos comercios se encon- 
traban en áreas con cierto grado de restricción (unidades habitacionales, calles cerradas) donde la población ajena es fácil de identificar y provoca cierta desconfianza al expendedor.

Cabe señalar que, ante la venta de cigarros sueltos, las compañías tabacaleras al ver afectados sus intereses - con esta venta las ganancias del comerciante son mayores pero las tabacaleras venden menos-, en una estrategia de aparente apoyo al combate contra el tabaquismo, apoyan la prohibición de venta por unidades y en cajetillas menores de 14 cigarros, misma que desde 1999 se había publicado en el Diario Oficial de la Federación del 9 de agosto.

La accesibilidad de los cigarros dentro de los comercios se da básicamente a través de los expendedores; sin embargo, en los puestos ambulantes o semifijos en más de la mitad de los casos (52.1\%) los cigarros - que en este tipo de comercios son sueltos-, fueron tomados directamente por el comprador. En comercios como las tiendas, las farmacias, las vinaterías y las papelerías, los cigarros generalmente los despacha el dependiente; cuando son sueltos, éste acerca la cajetilla abierta al comprador para que tome las unidades que desee. En los comercios de autoservicio los cigarros siempre se obtuvieron a través del despachador.

Para los menores de edad la accesibilidad no varió mucho; el mayor número de veces los despachó el dependiente y, de las 20 ocasiones que los obtuvieron por autoservicio, cinco ( $25 \%$ ) fueron en tiendas, en ventana o en miscelánea y $15(75 \%)$ en puestos ambulantes.

Durante la dinámica de la transacción, en lo referente al cumplimiento de la disposición oficial de solicitar una identificación, el número de expendedores que cumplieron fue muy pequeño (sólo ocho casos); de igual forma lo fueron el preguntar la edad del menor (15 ocasiones) o para quién eran los cigarros (31 ocasiones).

En las pocas ocasiones en que se cumplieron estas disposiciones no se garantizó en forma total la no venta a los menores, al observarse que en tres de las ocho ocasiones en que se solicitó la identificación les vendieron los cigarros. De igual forma, no negaron el producto $60 \%$ de los que preguntaron la edad y $87 \%$ de los que preguntaron para quién eran los cigarros. Lo anterior hace pensar que para los expendedores dichas disposiciones carecen de importancia, comparadas con la posibilidad de lograr una venta.

$\mathrm{Al}$ analizar las características de los menores según el sexo, se encontró lo informado por autores como Kuri, Altman, Wakefield, Foster, Erickson:,111-14 las niñas tienen menos dificultad para comprar cigarros que los niños, aunque difieren de otros que no hallaron diferencias por sexo. ${ }^{15,16}$ En el presente estudio, las mujeres tuvieron un poco más de dificultad para obtener los cigarros que los hombres, sin embargo esto pudo ser consecuencia de que en esta investigación el número de intentos por sexo no fuera el mismo.

El análisis de la edad de los menores en relación con el resultado de la venta de cigarros tampoco presentó resultados que apoyaran lo informado por Altman, Wakefield, Foster, y Andrews, ${ }^{11-13,17}$ donde se observó que conforme el menor tenía más años, la dificultad para comprar cigarros era menor. En esta investigación la edad no registró un aumento proporcional al porcentaje de éxito en la compra de cigarros, debido a que en las edades extremas (10 y 14 años) fue donde se registró el mayor número de intentos exitosos, con un comportamiento irregular en el resto de las edades.

Partiendo de las hipótesis en las cuales se propone que existe algún tipo de asociación entre las características de los expendedores y la venta a menores, al analizar los resultados encontramos que únicamente el sexo del expendedor registró un cierto grado de asociación $(R M p=2.04)$; cuando el expendedor fue hombre, representó un mayor riesgo para vender cigarros a menores que cuando fue mujer; la diferencia entre la proporción de venta, aunque pequeña, es significativa.

En relación con el resto de variables referentes al expendedor, ninguna presentó asociación con la venta de cigarros a menores en forma directa (análisis bivariado). Se evaluó entonces alguna posible confusión y se estratificó el análisis por las posibles variables confusoras; sin embargo, aun así no existió asociación. Se procedió entonces a realizar el análisis multivariado, y se encontró que el modelo propuesto (cuadro V) es el que mejor explica la asociación entre las variables involucradas en la venta de cigarros a menores.

Durante el desarrollo del estudio, se hicieron evidentes aspectos que no se pueden dejar de lado. Por una parte, se registró un alto porcentaje de venta de cigarros sueltos, que significa una violación a la legislación vigente que establece que cajetillas de 14 cigarros es el mínimo permitido; otro aspecto fue la venta a menores y la falta de cumplimiento en la solicitud de su identificación al comprador; sin embargo, los expendedores ignoran estos aspectos, pues saben que la vigilancia del cumplimiento de las disposiciones oficiales es casi nula y además, cuando se llega a identificar alguna violación a la legislación vigente, es muy probable que no se apliquen las sanciones correspondientes, debido al alto índice de corrupción que impera en nuestro país.

Por otro lado, también se identificó en los comercios a menores trabajadores. Aunque en la mayoría de las ocasiones fue en comercios habilitados en ventanas de casas habitación, no deja de llamar la atención que también se registraron en comercios semifijos o ambulantes. 
Por lo anterior, es obvio que modificar la legislación para restringir la venta a menores no funciona si no se vigila estrechamente su cumplimiento, y prueba de ello es que a pesar de las modificaciones a la legislación respecto al control de venta de cigarros a menores de edad, el porcentaje encontrado $(73 \%)$ indica que no se ha observado una disminución sustancial respecto al registrado en 1997 (79\%), lo que hace evidente que esta estrategia por sí sola no es efectiva.

Ante esta situación es necesario que en México, apoyados en el hecho de que en agosto del 2003, a través del comunicado de prensa No. 172 del día 12, se informó que nuestro país firmó el Convenio Marco para el Control del Tabaco, mismo que deberá ser ratificado por el Senado de la República para adquirir carácter de ley, se fortalezcan las medidas que han mostrado tener mayor impacto en la reducción o en el retraso del inicio en el consumo de productos de tabaco - especialmente cigarros-como son:

- El aumento gradual del impuesto al tabaco (como medida de Salud Pública);

- el empleo de los fondos generados por impuestos al tabaco en programas preventivos y de rehabilitación;

- la ampliación del tema de tabaquismo en los libros de texto de primaria y su incorporación en los de secundaria, $\mathrm{y}$

- la creación de una licencia especial para comerciar productos de tabaco (similar a la de las bebidas alcohólicas).

Sin embargo, es obvio que los cambios que se puedan generar con el trabajo educativo y legal en la venta de cigarros a menores de edad, y en consecuencia en la disminución del tabaquismo, especialmente entre los adolescentes, es algo que no se puede valorar en forma inmediata ni en un contexto individual. Por lo anterior, es necesario mantener una vigilancia continua del problema y evaluar a mediano plazo si las acciones tienen un efecto directo en la disminución del problema de la venta de cigarros a menores.

\section{Referencias}

I. Kuri P, Cravioto P, Hoy M, Huerta S, Revuelta A, Tapia CR. Illegal sales of cigarettes to minors. Morb Mortal Wkly Rep 1997;46 (20):440-444.

2. Secretaría de Salud. Encuesta Nacional de Adicciones. México:

Dirección General de Epidemiología, 1998.

3. Secretaría de Salud. Programa contra el tabaquismo. México: Consejo Nacional Contra las Adicciones, 1992.

4. Center for Disease Control and Prevention Community. Illegal sales of cigarettes to minors-Ciudad Juárez, México; El Paso Texas and Las Cruces New México, 1999. Morb Mortal Wkly Rep 1999; 48(19):394-398.

5. Kleinbaum GD. Logistic regression: a self-learning text. Nueva York: Springer-Verlang, 1994.

6. Hosmer WD, Lemeshow S. Applied logistic regression. Nueva York: John Wiley and Sons, 1989.

7.Visuta VB. Análisis estadístico con SPSS para Windows. En: Estadística multivariante. México: McGraw-Hill, 2000; vol 2.

8. Voorhees CC, Swank RT, Stillman FA, Harris HW. Cigarettes sales of african-american and white minors in low-income areas of Baltimore. Am J Public Health 1997; 87(4):652-654.

9. O'Grady B,Asbridge M,Abernathy T.Analysis of factors related to illegal tobacco sales to young people in Ontario. Tob Control 1999; 8:30I-305.

I0. Difranza J, Savageau J,Aisquith BF. Youth access to tobacco: the effects of age, gender, vending machine locks and "it's the law" program. Am J Public Health 1996; 86(2):22I-224.

I I. Altman DG, Foster V, Rasenick-Dous L, Tye JB. Reducing the illegal sale of cigarettes to minors. JAMA 1989;261:80-83.

12. Wakefield M, Carrangis J, Wilson D, Reynols C. Illegal cigarette sales to children in south Australia. Tob Control 1992; I: I I4- I 7.

13. Foster JL, Hourigan M, McGovern P. Avialability of cigarettes to underage youth in three communities. Prev Med 1992; 21:320-328

14. Erickson AD, Woodruff SI, Wildey MB, Kenney E. Baseline assessment of cigarette sales to minors in San Diego Contry, California. J Community Health 1993; 18:213-224.

15. Skretny MT, Cummings M, Sciandra R, Marshall J. An intervention to reduce the sale of cigarette to minors, NY State. J Med 1990; 90:54-55. 16. Center for Disease Control and Prevention Community. Minor's access to tobacco Missouri 1992 and Texas 1993. Morb Mortal Wkly Rep 1993; 42:125-128.

17. Andrews B, Mckay E, Hann A, Stephenson J. Cigarettes sales to juveniles: retrailer compliance in Dubbo, NSW. Health Promot J Austr 1994; 4:13-17. 